\title{
Assessment of personal exposure to air pollutants in Scotland - an integrated approach using personal monitoring data
}

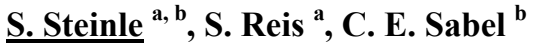 \\ ${ }^{a}$ Centre for Ecology \& Hydrology Edinburgh, Bush Estate, Penicuik, Midlothian, EH26 OQB \\ ${ }^{b}$ Geography, College of Life \& Environmental Sciences, University of Exeter, Amory Building, Rennes Drive, \\ Exeter, EX4 4RJ \\ Email: susein@ceh.ac.uk
}

\begin{abstract}
High levels of air pollution are associated with adverse effects on public health. Pollutant concentrations are typically subject to a high spatial and temporal variability. To investigate and quantify potential relations between pollutant concentrations and health effects, e.g. cases of respiratory diseases, sophisticated geospatial tools and methods are required.
\end{abstract}

Air pollutants are ubiquitous and a certain level of exposure is inevitable. For risk assessments and public health advice, however, it is necessary to quantify human exposure to specific pollutants of concern. This is a challenging task as individual daily mobility patterns substantially influence exposure to air pollutants over time and in space. But it is not only people's activities making the quantification difficult, also air chemistry, microclimatic and meteorological influences are changing over space and time, resulting in high spatial and temporal variation of ambient pollutant concentrations.

Within a research project funded by the Scottish Government (EDPHiS, Environmental Determinants of Public Health in Scotland), the application of GIS methods and tools for integrating data from personal monitoring trials with supporting Scotland-wide datasets such as air pollution concentrations, land use and population data for personal exposure assessment will be examined.

The work described here is conducted in the frame of a joint PhD studentship between the Centre for Ecology \& Hydrology and the University of Exeter. It focuses on the development of methods for personal exposure monitoring as well as the integration of measured data with supporting secondary data for improving human exposure assessment. For this purpose, an experimental design with a small, wearable personal monitoring device to derive personal time-activity patterns and exposure profiles is currently devised. Resulting personal exposure profiles will be integrated and assessed using geographic Information Systems (GIS) methods for a complementary human exposure assessment approach.

The work presented here will focus on the aspect of monitoring personal activity and resulting exposure. Its challenges and methods for quantification will be elaborated.

Key Words: Air pollution, GPS, personal exposure monitoring, public health, Scotland 


\section{INTRODUCTION}

Human exposure to air pollutants and environmental influences in general is a highly topical issue. Increasing mobility and economic status result in increasing traffic and energy consumption and therefore high ambient levels of chemical compounds or so-called air pollutants such as Nitrogen Oxides $\left(\mathrm{NO}_{\mathrm{x}}\right)$, Carbon Monoxide $(\mathrm{CO})$, Particulate Matter (PM) and ground level Ozone $\left(\mathrm{O}_{3}\right)$. Urban areas with high population densities and high traffic flows are especially affected. Air pollutants are ubiquitous and a certain level of exposure is inevitable whether a person is indoors or outdoors. For risk and impact assessments of air pollution control policies such as the National Air Quality Strategy, the Local Air Quality Management or the Road Vehicles Regulations (Environmental Protection UK, 2011), it is necessary to quantify human exposure to air pollution. The challenge, however, is that human exposure is a function of concentration and time (Nuckols et al., 2004) and thus is not straightforward to quantify.

To analyse personal exposure the actual ambient concentration levels of the pollutant of concern are needed with sufficient temporal and spatial resolution. Recent developments in GPS technology allow monitoring a person's individual activity patterns, and thus exposure to a specific pollutant, while moving in space and time. The advantage of personal exposure profiles is that the actual concentration levels are measured. This is a highly localised approach which is suitable to assess short term effects of air pollution. For further analysis this monitoring data needs to be integrated with temporal and spatially aggregated concentration data as well as with land use data to derive conclusions about associations between concentration levels, the environment the person is moving in and potential health impacts.

\section{RESEARCH BACKGROUND}

In the following the term personal exposure is defined and related topics explained. A cause-effect model which reflects relationships between environment and human system is introduced. The model is used to help placing the area of this $\mathrm{PhD}$ research in the context of environment and health.

\subsection{Personal exposure to air pollutants}

Personal exposure can be defined as "...the event during which a person comes into contact with a pollutant" (Ott, 1985). More specifically, Branis (2010) defines personal exposure as the measurement of a pollutant of concern performed by a monitor or sampler which is worn at a point near the breathing zone of a person while sampling. According to Nieuwenhuijsen (2000) the breathing zone from where air is inhaled is within $30 \mathrm{~cm}$ of the nose and mouth. Personal exposure includes pollutants from outdoor and indoor air as well as pollutants generated by the person's activities itself. The latter also is subject to the so called personal cloud effect which describes the fact that the exposure measured by a personal monitor is higher than what is predicted by the indoor concentration measured by a stationary indoor station (Gerharz, 2009, Wu, 2005, Ferro et al., 2004, Colbeck and Nasir, 2010, Branis, 2010). For this study however, the personal cloud effect is not considered.

Personal exposure takes place when the pollution concentration at a particular place and time is above zero, and the person is present in that same place and at that time. However, this does not necessarily mean that the person receives a dose (i.e. the amount of a pollutant that actually enters the human body). Eventually this means, that exposure can be without a dose, but there can never be a dose without exposure (Ott, 1985). Exposure can also be integrated over time to incorporate the duration of exposure. The spatial concentration field is a function of time as well, thus it will change as the person moves through space (Ott, 1982).

\subsection{Cause-effect relationship - from state to exposure}

For risk assessment and Health Impact Assessment (HIA), models which reflect the relations between the environmental and the human system are commonly used. The idea behind such cause-effect relationship models is to conduct impact pathway analyses, which means structuring and simplifying working with the complex interactions between environmental and socio-economic factors (EEA, 2007).

A conceptual model for a strategic approach to assess environmental effects on health (Morris et al., 2006) is the modified DPSEEA model (Drivers, Pressures, State, Exposure, Effects and Actions) shown in Figure 1. In comparison to the standard model, the modified DPSEEA model uses "Context" additionally. Context is introduced as a "bubble" around "Exposure" and "Effect" in a way that the transition from environmental state (i.e. ambient concentrations) to exposure and effects includes contextual issues such as social, economic, behavioural or demographic factors. By representing external influences, context is crucial for personal exposure assessment as it includes, among other things, personal living conditions which determine someone's characteristic individual exposure. 


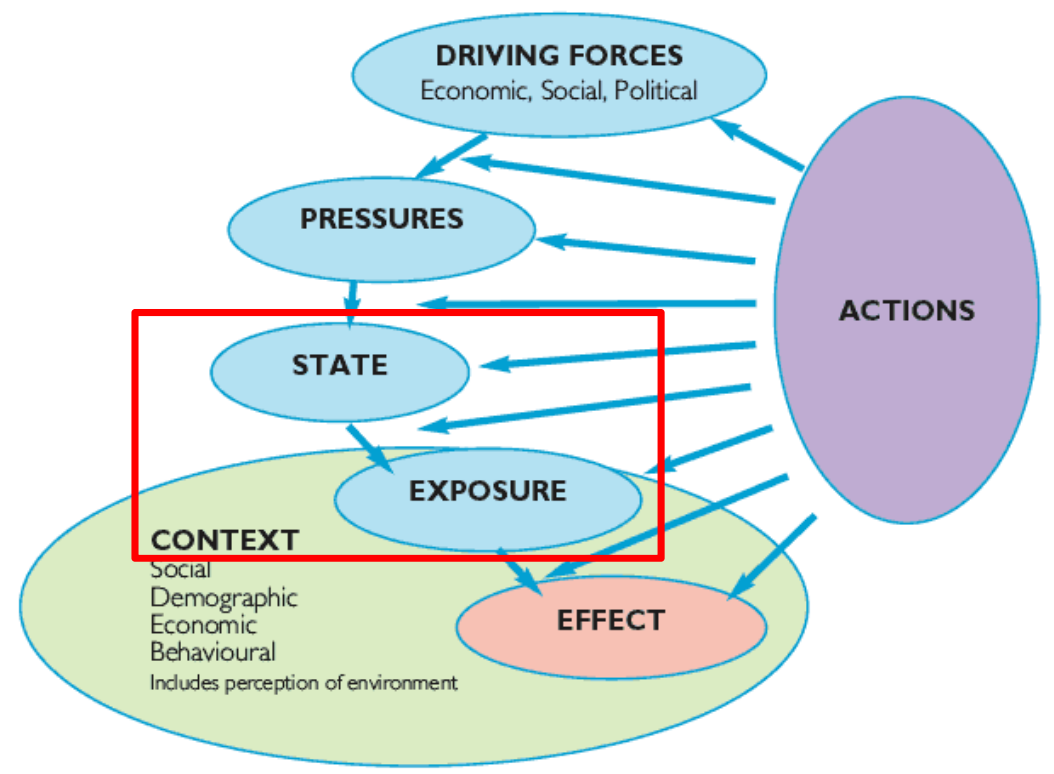

Figure 1 - The modified DPSEEA model (http:/www.scotland.gov.uk/Publications/2008/12/11090318/7;

$(20 / 05 / 2011))$

The modified DPSEEA model provides a simple illustration "...of the way in which the environment influences health and how environmental state is influenced by higher causes" (Morris et al., 2006). Its linear structure represents a hierarchical chain of causation which allows mapping actions or interventions directly on to the chain. "Actions" can influence all other parts of the model through policies or regulations which aim to minimise emissions for instance or through societal response. According to Morris et al. (2006) the modified DPSEEA model has its application particularly in community planning with targets like improving health and wellbeing or greater equality in health.

The focus of the work described here is on the elements "State", which in this case is the ambient concentration of an air pollutant of concern, and "Exposure" which represents the individual human exposure to this specific air pollutant, as well as the relationship between them.

\section{RESEARCH OUTLINE}

The scope of our work is to understand more about the associations between air pollution concentration and personal exposure to them in connection to time-activity. Therefore we have to better understand the variation of the respective pollutants over space and time. The first step of this research project is to develop a method for personal monitoring of individuals in Scotland. Since people move frequently in space and time and show individual social behaviour the hypothesis is, that depending on environment and lifestyle, exposure to air pollution is significantly different between individuals.

Personal exposure monitoring offers the possibility to observe and quantify small area changes in the environment over time regarding exposure to and impact of ambient air pollution levels. A better understanding of the exposure process to certain air pollutants and the potentially resultant impacts on human health can help design more efficient sampling and monitoring strategies for future studies and improving HIA with regard to air pollution. The derived individual exposure - concentration profiles will be:

- Compared with measurements from fixed monitoring sites as well as with modelled concentration data. Here the aim is identifying a method (or methods respectively) which proves to be the most accurate, effective and practicable one for assessing personal and groups exposure to pollutants and potential health effects

- Integrated with land cover data for identifying potential associations between land cover type and changes in concentration levels 
- Integrated with measured and modelled concentration data, land cover data, population data and respiratory disease indicators for identifying potential associations between air pollutant concentration exposure - respiratory disease

\section{DERIVING PERSONAL EXPOSURE USING GPS MONITORING}

The study area for this research project is Scotland, a country with highly heterogeneous environments. The Central Belt between Edinburgh and Glasgow is densely populated with a high level of infrastructure, whereas large parts of the country are rural, have significantly less population and hence infrastructure and industry (Figure 2).
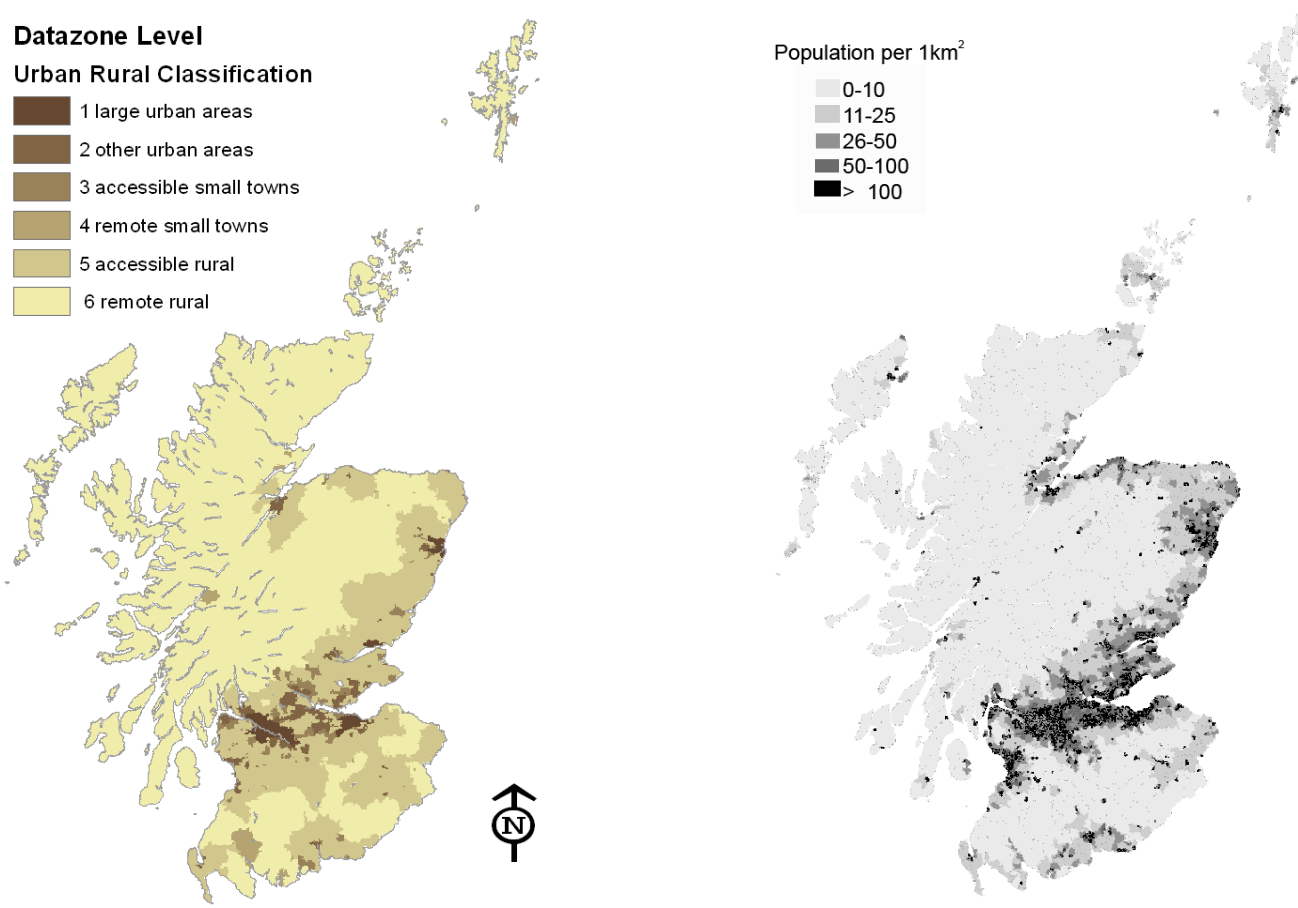

Figure 2. Scottish Government urban rural classification $2009 / 10$ (left) and population density $(1 \times 1 \mathrm{~km})$ based on 2009 midyear estimates (right) on data zone (DZ) level ${ }^{1}$

It is a fact that all exposure estimates based on surrogate measurements such as point measurements of environmental pollution or pollution models for instance, imply a rough approximation of actual exposure. This is because these methods for deriving exposure estimates do not take direct account of people moving in space, i.e. within the pollution field, and time (Briggs, 2000). With a personal monitoring device, however, the actual concentration a person is exposed to can be measured in real-time- actual exposure. By deriving concentration exposure profiles mainly in the south-eastern part of the country, including urban and rural areas, the differences in air pollution concentration and thus exposure to it can be assessed.

In cooperation with Heriot-Watt University in Edinburgh a custom-made monitoring device has been designed and a prototype is currently being produced. This device includes sensors to measure $\mathrm{NO}_{2}$ as a reactive and $\mathrm{CO}$ as a non-reactive indicator for complex pollutant mixes. The decision to monitor these two pollutants result from the availability of small and lightweight sensors as well as the role of the pollutants as indicators for pollutant mixes and potential health effects. Also integrated in the device are Wi-Fi and Bluetooth sensors plus a GPS receiver for geolocation logging. The combination of the three different technologies for tracking is hoped to improve logging location, especially indoors where people in industrialised countries spend about $85 \%$ of their time (Lazaridis and Colbeck, 2010). The device will also include sensors for humidity and temperature. These two indicators can provide useful information on the Microenvironments (MEs) people move in, especially on housing quality with regard to dampness and mould which can affect human health. The design of this device

1 Data zones: geographical units with an approximate population between 500 and 1000, excluding people not living in households. Data zones nest within Council Areas and as far as possible meet the criteria of compactness, homogeneity and respect other boundaries e.g. physical environment. There are 6,505 Data Zones in Scotland. 
has been done with the aim of developing a complementary approach as it gives information on the pollutant concentration, the duration and frequency of exposure as well as the pattern of exposure. Although this information can be logged with the device, time-activity-diaries (TAD) and questionnaires are necessary for quality control and additional information such as socio-economic background of the monitored person. An exposure study without this information gained by the use of TAD and questionnaires is according to Briggs (2000) inherently deficient.

It has to be kept in mind that this experiment will be a pilot study with limited extent regarding the number of participants and the area of Scotland covered, but it is set up to give a first insight of the application of novel, lightweight, integrated sensors. Since we want to measure every person's exposure over a maximum of a week's time, the number of profiles is limited further. Deriving profiles over a week has the advantage of detecting differences between weekdays and weekend as well as differences in small area changes when the daily routine is changed (for instance going to a meeting in a different part of town instead of the normal office working day). Additionally measurements over a week's time should derive enough data which is useable, since it is expected that people behave differently during the first day(s) of carrying the monitor. In general it is aimed to derive profiles from "classic" weeks. However, we also want to take opportunities to gain special profiles from people going on holiday for example, this way we are hoping to derive profiles from different areas in Scotland.

The aim is to cover the difference in pollution concentration between urban and rural areas with these profiles. $\mathrm{NO}_{2}$ is produced by all high temperature combustion processes, such as electric power stations and other industrial sites which are stationary sources. It is aimed to include different modes of transport in the profiles since traffic is the most significant mobile source of $\mathrm{NO}_{2}$. The second pollutant of interest, $\mathrm{CO}$, is formed by incomplete combustion processes with road traffic again as the largest source (DEFRA, 2011). It is expected to detect differences in exposure between pedestrians, cyclists, users of public transport or car drivers. Such differences have for instance been identified for exposure to $\mathrm{PM}_{2.5}$, ultrafine particle counts and $\mathrm{CO}$ by Kaur et al. (2005) within the DAPPLE project in London (www.dapple.org.uk). Indoor sources of $\mathrm{NO}_{2}$ and $\mathrm{CO}$ are for instance gas appliances for cooking and heating especially when they are badly installed or maintained and tobacco smoke (Department of the Environment, 1994, WHO, 2010, Flachsbart, 2007).

Monitoring the concentration levels in indoor environments allows us to measure the spatio-temporal variations of $\mathrm{NO}_{2}$ and $\mathrm{CO}$ concentrations and helps identifying sources of both pollutants. Indoor pollutant concentrations depend on the source such as heating system or gas oven as well as on the ventilation type or mode of ventilation since indoor air is a mixture of in- and outdoor air. Here it is expected to detect seasonal differences due to heating over cold periods and potentially less ventilation than in warmer months. To get an idea of these seasonal differences in the pollutant concentrations it would be good to get profiles from different times of the year. However, the gap between the two measurements (ideally the same person and their classic environments twice) should not be too long since we have to wait for the data.

The participants will also be asked to fill in a questionnaire to provide additional information on socioeconomic factors such as housing type, number of people living in the household, profession, information on workplace, transport mode, weather conditions etc., but also on general information such as age, gender and family status. Additionally the participants will have to fill in a short TAD once a day where they are asked to recall the day's activities to support the data derived from the monitor.

\section{DATA INTEGRATION AND ANALYSIS}

The overall aim of data integration and analysis is to identify or give advice on which method(s) for assessing human exposure to air pollutants prove to be the most accurate, effective and practicable one(s). Moreover, integration allows linking the state of the physical environment with socioeconomic factors and health impacts with the aim of improving health impact and risk assessment.

Complete concentration - exposure profiles will be compared with other available concentration data such as data from fixed monitoring sites or modelled background maps. Advantages/disadvantages for the respective method such as modelled vs. measured, individual actual exposure vs. modelled full spatial coverage will be discussed. The advantage of using the novel device is that complementary datasets including time, location and concentration will be derived. This is an improvement compared to analyses where time and location have to be integrated with concentration data from other sources as shown in Figure 3. In this example time and location have been derived with a GPS receiver, the $\mathrm{NO}_{2}$ concentration is the projected annual mean background concentration for 2010 from www.scottishairquality.co.uk (accessed 07/06/2011). In this case a time-activity pattern has been linked with temporal and spatial aggregated data that has no real connection to the individual profile. So, Figure 3 is not showing actual exposure. 


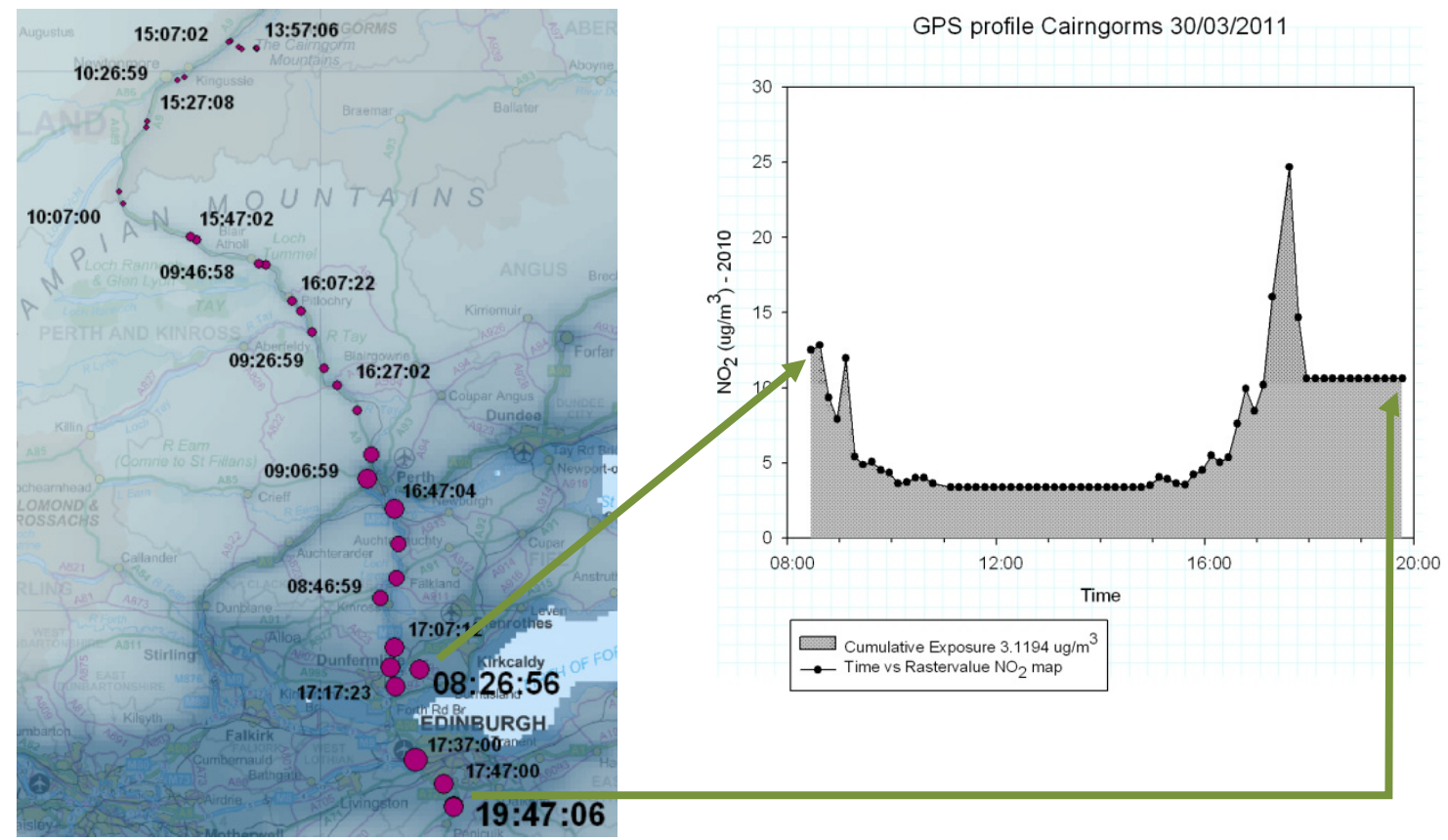

Figure 3. Integrating a GPS profile (time and location) with the projected annual background $\mathrm{NO}_{2}$ concentration. The above approach of integrating movement patterns with static concentration levels is not as yet analysing actual exposure, but quantifies the potential exposure over time.

The profiles will also be integrated in GIS with land cover data to analyse the differences between the physical environments people have been moving in and concentration levels. Other potential associations will be discussed, for instance between ambient concentrations and land cover type or housing quality. Integration of exposure data with the Scottish Index of Multiple Deprivation (SIMD) will be done for identifying potential associations between exposure and multiple deprivation. Integrating health indicators such as the number of asthma inhalers prescribed per General Practitioner (GP) or pharmacy helps to identify potential associations between air pollution and health. It is expected to quantify differences in exposure between urban and rural areas.

Initially we intend to hand out the device to colleagues from whom we have classic GPS profiles from day trips around Edinburgh and the Cairngorm national park to highlight differences in monitoring methods. These GPS profiles have been derived to test the practicability of the receiver for tracking people's movements in their everyday environment. Problems detected were signal blockage and interference near or inside buildings, especially in urban areas as well as in cars. Also the GPS receiver used needed a relatively long time to reestablish a connection to the satellites when the signal has been interrupted. Hence we concluded that a GPS receiver alone is not sufficient for our experiment. We therefore aim to integrate a Wi-Fi receiver in the monitoring device to enable locating people indoors. Further on we want to discuss and compare the possibilities of analysis depending on the available input data (Figure 3).

\section{DISCUSSION AND CONCLUSION}

Applying a newly developed personal device for monitoring exposure to $\mathrm{NO}_{2}$ and $\mathrm{CO}$ will help improve monitoring strategies and HIA. A better understanding of spatiotemporal changes in concentration levels and their relations to specific environments and sources can support decision making with regard to emission control policies and public health protection.

This novel monitoring approach allows analysis of the complex relations between pollutant concentrations, exposure and health impacts in a different way. The comprehensive measurement of all three components time, location and concentration - allows the determination of an individual's actual exposure to the specific pollutant while moving in space and time. By integrating the profiles with health indicators and socioeconomic data, associations between the physical and socioeconomic environment and health impact can be detected. This approach can be extended to other pollutants of concern depending on the availability of small sensors that can be integrated into the device. 
The next step after individual exposure assessment at high spatial and temporal resolution, is developing and applying methods and tools for exposure assessment for larger populations. This up-scaling is necessary to assess exposure to air pollution and potential health effects for a population, while potentially reducing the need for intensive personal monitoring field trials, which are both time and cost intensive.

\section{ACKNOWLEDGMENTS}

This work is supported by the project Environmental Determinants of Public Health in Scotland (EDPHiS), funded by the Research Environment and Rural Affairs Department (RERAD) of the Scottish Government, and the LIAISE Network of Excellent, funded under the 7th Framework Programme of the European Commission.

\section{REFERENCES}

Branis, M. (2010). Personal Exposure Measurements. In Lazaridis, M., Colbeck, I. (Eds.) Human Exposure to Pollutants via Dermal Absorption and Inhalation, 97-141.

Briggs, D. J. (2000). Exposure assessment. In Elliott, P., Wakefield, J. C., Best, N. G. \& Briggs, D. J. (Eds.) Spatial Epidemiology. Methods and Applications, 335-359.

Colbeck, I. \& Nasir, Z. A. (2010). Indoor air pollution. In Lazaridis, M., Colbeck, I. (Eds.) Human Exposure to Pollutants via Dermal Absorption and Inhalation. 41-72.

DEFRA (2011). What are the causes of air pollution. $\underline{\text { http://uk- }}$ air.defra.gov.uk/documents/What are the causes_of_Air_Pollution.pdf. (Accessed 19/07/2011)

Department of the Environment (1994). Expert Panel on Air Quality Standards. Carbon Monoxide EEA (2007). The DPSIR framework used by the EEA. Copenhagen, European Environment Agency (EEA). Environmental Protection UK (2011). Air Pollution Laws.

Ferro, A. R., Kopperud, R.J. \& Hildemann, L. M. (2004). Source strengths for indoor human activities that resuspend particulate matter. Environmental Science and Technology, 38, 1759-1764.

Flachsbart, P. G. (2007). Exposure to Carbon Monoxide. In Ott, W. R., Steinemann, A. C. \& Wallace, L. A. (Eds.) Exposure Analysis. 113-146.

Gerharz, L., Krueger, A., Klemm, O. (2009). Applying indoor and outdoor modeling techniques to estimate individual exposure to PM2.5 from personal GPS profiles and diaries: A pilot study. Science of the Total Environment, 407, 5184-5193.

Kaur, S., Nieuwenhuijsen, M. \& Colvile, R. (2005). Personal exposure of street canyon intersection users to PM2.5, ultrafine particle counts and carbon monoxide in Central London, UK. Atmospheric Environment, 39, 3629-3641

Lazaridis, M. \& Colbeck, I. (2010). Environmental Levels. In Lazaridis, M., Colbeck, I. (Eds.) Human Exposure to Pollutants via Dermal Absorption and Inhalation. 1-40.

Morris, G. P., Beck, S. A., Hanlon, P. \& Robertson, R. (2006). Getting strategic about the environment and health. Public Health, 120, 889-903.

Nieuwenhuijsen, M. J. (2000). Personal exposure monitoring in environmental epidemiology. In Elliott, P., Wakefield, J. C., Best, N. G. \& Briggs, D. J. (Eds.) Spatial Epidemiology. Methods and Applications, 360-374.

Nuckols, J. R., Ward, M. H. \& Jarup, L. (2004). Using geographic information systems for exposure assessment in environmental epidemiology studies. Environmental Health Perspectives, 112, 1007-1015.

Ott, W. R. (1982). Concepts of human exposure to air pollution. Environment International, 7, 179-196.

Ott, W. R. (1985). Total human exposure. Environmental Science and Technology, 19, 880-806.

WHO (2010). WHO guidelines for indoor air quality: selected pollutants.

Wu, C.-F., Delfino, R. J., Floro, J. N., Quintana, P. J. E., Samimi, B. S., Kleinman, M. T., Allen, R. W., Sally Liu, L.-J. (2005). Exposure assessment and modeling of particulate matter for asthmatic children using personal nephelometers. Atmospheric Environment, 39, 3457-3469. 